\title{
Biological and clinical impact of hemangioblastoma-associated peritumoral cysts in von Hippel-Lindau disease
}

\author{
Kristin Huntoon, PhD, DO,,2 Tianxia Wu, PhD, ${ }^{3}$ J. Bradley Elder, MD, ${ }^{1}$ \\ John A. Butman, MD, PhD, ${ }^{4}$ Emily Y. Chew, MD, ${ }^{5}$ W. Marston Linehan, MD, ${ }^{6}$ \\ Edward H. Oldfield, MD, ${ }^{2,7}$ and Russell R. Lonser, MD ${ }^{1,2}$
}

'Department of Neurological Surgery, The Ohio State University Wexner Medical Center, Columbus, Ohio; ${ }^{2}$ Surgical Neurology Branch and ${ }^{3}$ Office of the Clinical Director, National Institute of Neurological Disorders and Stroke; ${ }^{4}$ Neuroradiology Section, Diagnostic Radiology, Clinical Center at the National Institutes of Health; ${ }^{5}$ Division of Epidemiology and Clinical Applications, National Eye Institute; ${ }^{6}$ rologic Oncology Branch, Center for Cancer Research, National Institutes of Health, Bethesda, Maryland; and 'Department of Neurological Surgery, University of Virginia Health Sciences Center, Charlottesville, Virginia

OBJECTIVE Peritumoral cysts are frequently associated with CNS hemangioblastomas and often underlie neurological morbidity and mortality. To determine their natural history and clinical impact, the authors prospectively analyzed hemangioblastoma-associated peritumoral cysts in patients with von Hippel-Lindau (VHL) disease.

METHODS Patients with VHL disease who had 2 or more years of follow-up and who were enrolled in a prospective study at the National Institutes of Health were included. Serial prospectively acquired laboratory, genetic, imaging, and clinical data were analyzed.

RESULTS One hundred thirty-two patients (of 225 in the VHL study with at least 2 years of follow-up) had peritumoral cysts that were followed for more than 2 years (total of 292 CNS peritumoral cysts). The mean age at study entrance was $37.4 \pm 13.1$ years ([mean \pm SD], median 37.9, range 12.3-65.1 years). The mean follow-up was $7.0 \pm 1.7$ years (median 7.3, range 2.1-9.0 years). Over the study period, 121 of the 292 peritumoral cysts (41.4\%) became symptomatic. Development of new cysts was associated with a larger number cysts at study enrollment $(p=0.002)$ and younger age $(p<0.0001)$. Cyst growth rate was associated with anatomical location (cerebellum cysts grew faster than spine and brainstem cysts; $p=0.0002$ and $p=0.0008)$, younger age $(<35$ years of age; $p=0.0006)$, and development of new neurological symptoms $(p<0.0001)$. Cyst size at symptom production depended on anatomical location $(p<0.0001$; largest to smallest were found, successively, in the cerebellum, spinal cord, and brainstem). The most common location for peritumoral cysts was the cerebellum (184 cysts [63\%]; $p<0.0001)$.

CONCLUSIONS Peritumoral cysts frequently underlie symptom formation that requires surgical intervention in patients with VHL disease. Development of new cysts was associated with a larger number of cysts at study enrollment and younger age. Total peritumoral cyst burden was associated with germline partial deletion of the VHL gene.

http://thejns.org/doi/abs/10.3171/2015.4.JNS1533

KEY WORDS cyst; central nervous system; hemangioblastoma; natural history; treatment; von Hippel-Lindau disease; oncology

$\mathrm{T}$ HE most common von Hippel-Lindau (VHL) disease-associated neoplasms are hemangioblastomas of the CNS., Multiple craniospinal hemangioblastomas can be found in nearly $90 \%$ of patients with VHL disease. $5,6,9,16,17,20,21,23$ Although CNS hemangioblastomas are benign tumors, they can cause significant neurological morbidity and mortality due to mass effect caused by the tumor itself or, in many cases, an associated peritumoral cyst that forms adjacent to the tumor. ${ }^{1,4,26}$ Hemangioblastoma-associated peritumoral cysts form as plasma ultrafiltrate leaks through permeable tumor vessels into the surrounding CNS tissue. ${ }^{14}$ Prior studies have estimated that greater than $70 \%$ of hemangioblastoma-related neurological symptoms with sporadic occurrence and in VHL 
disease are more likely to be the result of the associated peritumoral cyst than the tumor itself. ${ }^{1,14,26}$

In spite of the frequent occurrence and deleterious effects of CNS hemangioblastoma-associated peritumoral cysts in VHL disease, their biological features are not fully defined. Consequently, their clinical impact and optimal management in VHL disease is not well understood. To develop deeper insights into their natural history, biology, and clinical management, we prospectively analyzed hemangioblastoma-associated peritumoral cysts using clinical, genetic, imaging, and laboratory data in a large series of patients with VHL disease.

\section{Methods \\ Patient Population}

Patients with VHL disease were included in a prospective study to determine the natural history of CNS tumors, including hemangioblastomas, in this disease. Informed consent was obtained from all patients before enrollment in this National Institutes of Health-approved protocol (NIH\#00-N-0140). ${ }^{11}$ The diagnosis of VHL disease was confirmed in study patients by using genetic testing and/or diagnostic clinical criteria..$^{13,19}$

\section{Germline Genotype Analysis}

The determination of germline mutations in the $V H L$ gene was achieved by peripheral blood sample analysis, as described previously. ${ }^{11,25}$

\section{Clinical and Imaging Assessment}

Patients were evaluated with neural axis imaging (MRI) and clinical examinations at approximately 6-month intervals, as described previously. ${ }^{11}$

\section{Cyst Characteristics}

To best assess cyst biology and clinical features, patients and peritumoral cysts with less than a 2-year followup were excluded. ${ }^{11}$ To avoid confounding information, imaging data analysis was terminated in patients and cysts at the initiation of systemic chemotherapy, stereotactic radiosurgery, or craniospinal radiation. ${ }^{2,24}$

MRI was used to calculate hemangioblastoma (T1weighted, after contrast addition) and associated cyst (T2-weighted) volume by a modified ellipsoid formula at each visit. ${ }^{15}$ Cyst growth patterns were classified as saltatory (growth and quiescent periods), linear, or exponential. Cysts were classified as stable if they did not progress in size. ${ }^{11}$

\section{Surgical Management}

Symptomatic hemangioblastomas were resected, as described previously. ${ }^{8,18,27,28}$ The peritumoral cyst walls were left intact during the removal of the associated hemangioblastoma(s).

\section{Statistical Analysis}

Patient characteristics and hemangioblastoma features were summarized using descriptive statistics. Peritumoral cysts with 2 or more years of follow-up and a minimum of
4 clinical and radiographic time points were used to study the cyst growth pattern. The cyst growth pattern was determined by mathematical characterization, as previously defined. ${ }^{11}$ Briefly, we applied a general linear model (SAS procedure GLM) to examine the association of patients' cyst burden (the total number of cysts) with age (categorized as less than 35 years, or greater than or equal to 35 years), sex, years of follow-up, and germline mutation type (partial deletion versus missense). This statistical model was also applied to assess the association of cyst burden (total number of cysts) and cyst development (new cyst formation during study period) with age, sex, total cyst number at study entrance, and germline mutation type.

Because most patients had more than 1 cyst, a general linear mixed model with the patient as random effect (procedure MIXED) was used to assess the association of growth rate with age, sex, symptoms, and cyst location. To assess for the accuracy of prediction of symptoms using cyst size, receiver operating characteristic (ROC) curve analysis was used. Survival analysis (Kaplan-Meier method) was performed using growth (cyst volume increased by at least $7.5 \mathrm{~mm}^{3}$ ) as an event of interest, and the stable cysts were referred to as censored. The time to event was defined as the time duration between the examination dates on which the cyst was detectable on imaging (12 $\mathrm{mm}^{3}$ ) to the examination date on which cyst enlargement was greater than $7.5 \mathrm{~mm}^{3}$. Because their distributions had a long right tail, quantitative outcome measures were logarithmically transformed. The difference of means based on transformed data were inverse transformed to fold. A $\mathrm{p}$ value of $\leq 0.05$ was used as significant. SAS version 9.2 was used for statistical analyses. The mean is expressed \pm SD throughout.

\section{Results}

\section{Patient Characteristics}

A total of 250 patients with VHL disease (accrual ceiling of protocol) were enrolled in the study. Two hundred twenty-five patients with 2 or more years of follow-up were included in the analysis. One hundred thirty-two (59\%) of these patients (63 males, 69 females) had hemangioblastoma-associated peritumoral cysts at some point during their assessment. The mean age at study entrance was 37.4 \pm 13.1 years (median 37.9, range 12.3-65.1 years). The mean follow-up was $7.0 \pm 1.7$ years (median 7.3, range $2.1-9.0$ years).

\section{Cyst Burden}

Over the course of the study, 292 peritumoral cysts were identified in the 132 patients. The cysts were located in the cerebellum (184 cysts; $63.0 \%$ of the total), brainstem (33 cysts; $11.3 \%$ ), spinal cord (71 cysts; $24.3 \%$ ), or supratentorial compartment (4 cysts; $1.4 \%$ ).

\section{Cyst Imaging Features}

Hemangioblastoma-associated peritumoral cysts appeared hypointense on T1-weighted postcontrast images and hyperintense on T2-weighted images. The cysts were hypointense on precontrast FLAIR MRI studies but hyperintense on postcontrast FLAIR MRI (Fig. 1). 

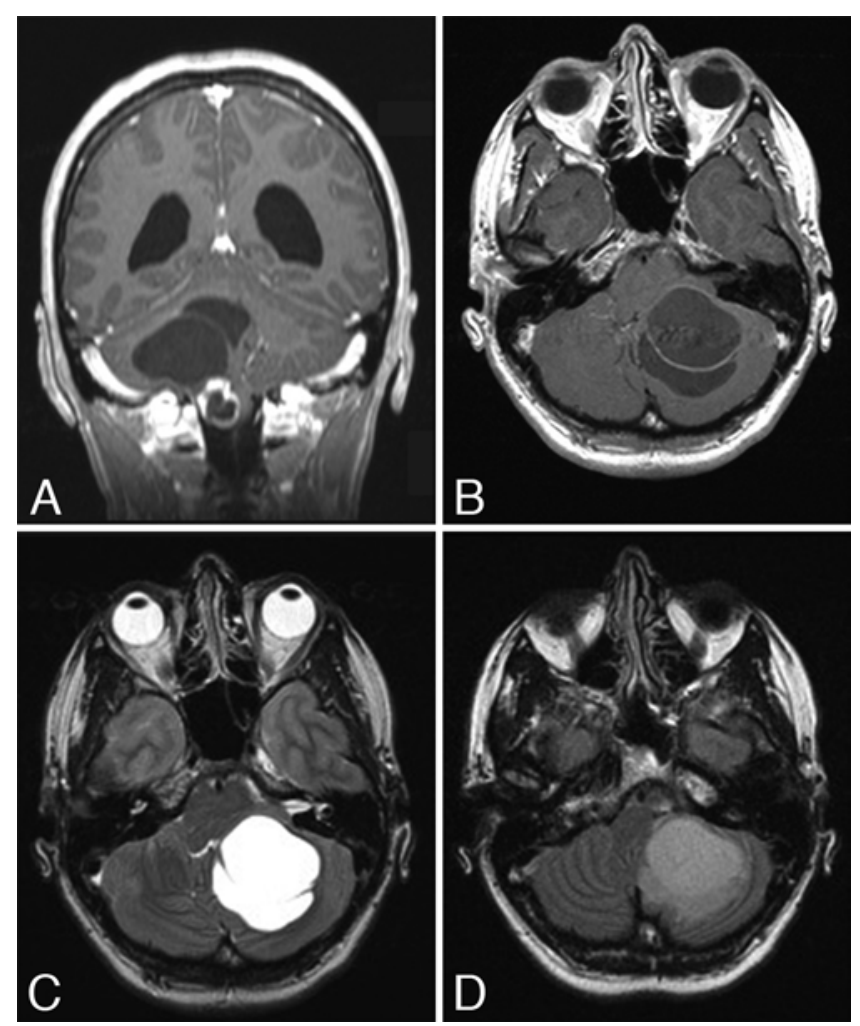

FIG. 1. Postcontrast MRI of a cerebellar hemangioblastoma with associated peritumoral cyst. The cyst emanates superiorly from the enhancing lesion and causes dilation of the fourth ventricle due to mass effect within the foramen magnum (A). This permits a unique side-by-side view of the cyst and ventricle seen on Gd-enhanced T1-weighted coronal and axial (A and B), T2-weighted axial (C), and FLAIR axial (D) images.

\section{Cyst Progression}

\section{Cyst Growth}

The incidence of progressive cyst growth depended on the interval of observation. Of the 187 at risk, 47 cysts (25\%) progressed in 0.6 years, 94 cysts $(50 \%)$ progressed in 1.5 years, and $141(75 \%)$ progressed in 3 years (Fig. 2). One hundred twenty-one cysts $(41.4 \%$ of all cysts, $76 \%$ of all hemangioblastomas-i.e., hemangioblastomas with or without a cyst) were associated with symptoms, and required resection of the causative hemangioblastoma (correlation of presence of cyst with symptom formation; $\mathrm{p}<$ 0.0001).

Cyst growth rate was associated with cyst location, age, and symptom. The mean cyst growth rate in the cerebellum was $1081.7 \pm 2732.1 \mathrm{~mm}^{3} /$ year (117 cysts; median $131.3 \mathrm{~mm}^{3} /$ year; range $0-19,040.2 \mathrm{~mm}^{3} /$ year; interquartile range [IQR] $695.0 \mathrm{~mm}^{3} /$ year). The mean cyst growth rate in the brainstem was $171.7 \pm 346.2 \mathrm{~mm}^{3} /$ year $(23$ cysts; median $12.1 \mathrm{~mm}^{3} /$ year; range $0-1282.6 \mathrm{~mm}^{3} /$ year; IQR $167.8 \mathrm{~mm}^{3} /$ year). The mean cyst growth rate in the spinal cord was $113.5 \pm 270.9 \mathrm{~mm}^{3} /$ year (47 cysts; median 15.5 $\mathrm{mm}^{3} /$ year; range $0-1615.6 \mathrm{~mm}^{3} /$ year; IQR $114.2 \mathrm{~mm}^{3} /$ year).

Cyst growth rate was associated with location (cerebellar cysts grew faster than spinal cord [4.6-fold] and brainstem cysts [5.8-fold]; $p=0.0002$ and $p=0.0008$ ). Cysts

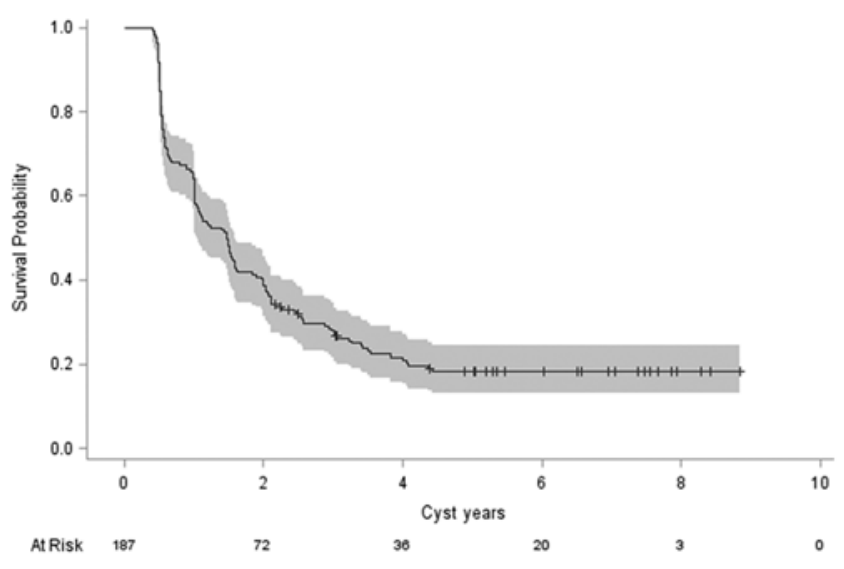

FIG. 2. Kaplan-Meier analysis of peritumoral cyst progression in patients with VHL disease over an 8-year period. Twenty-five percent (47 cysts) progressed in 0.6 years, approximately half (94 cysts) progressed in 1.5 years, and nearly three-quarters (141 cysts) progressed in 3 years.

in patients younger than 35 years of age grew 3.5-fold faster than those in patients 35 years or older $(p=0.0006)$. Cysts associated with symptoms grew 15.7-fold faster than asymptomatic cysts $(\mathrm{p}<0.0001)$.

\section{Pattern of Growth}

The pattern of peritumoral cyst growth was variable. Cysts (187 cysts had a 2-year follow-up in which a growth pattern could be determined) grew in a saltatory (78 cysts $[41.7 \%$ of cysts]), linear (18 cysts [9.6\%]), or exponential (51 cysts [27.3\%]) pattern. Thirty-eight cysts (20.3\%) remained stable over the observation period. Of the 60 symptomatic peritumoral cysts with more than 2 years of follow-up, 20 cysts $(33.3 \%)$ grew in a saltatory pattern, 27 cysts (45) grew exponentially, 11 (18.3\%) grew linearly, 1 cyst grew in an unknown pattern, and 1 cyst did not grow.

\section{Ratio of Cyst to Tumor Size}

Symptomatic cysts in the cerebellum had a significantly larger ratio of mean cyst size to corresponding tumor size (105.2 \pm 326.0 ; median 10.6; range 0.02-2047.4) compared with asymptomatic cerebellar cysts $(10.9 \pm 27.2$; median 1.4; range 0.01 to $18.5 ; \mathrm{p}<0.0001$ ). Symptomatic cysts in the brainstem had a significantly larger ratio of mean cyst size to corresponding tumor size of $7.0 \pm 10.6$ (9 cysts; range 0.06-31.6, median 1.6; IQR 11.9) compared with asymptomatic brainstem cysts ( 22 cysts; $4.8 \pm$ 11.9; range 0.02 to 56.7 ; median 1.0 ; IQR 4.3 ; $\mathrm{p}<0.0001$ ). Symptomatic cysts in the spinal cord had a significantly larger ratio of mean cyst size to corresponding tumor size of $13.8 \pm 18.5$ (range 0.03-59.5) compared with asymptomatic spinal cysts (mean $3.6 \pm 6.3$; range 0.08 to 36.2 ; p $<0.0001$ ).

Symptomatic cysts in the cerebellum (76 cysts) had 3.4 -fold ( $p<0.0001$ ) larger mean ratio of cyst size to corresponding tumor size compared with asymptomatic cerebellar cysts (90 cysts). Symptomatic cysts in the spine (23 cysts) had 2.5 -fold $(\mathrm{p}=0.0006)$ larger mean ratio of cyst size to corresponding tumor size compared with asymp- 
tomatic spinal cysts (48 cysts). As for brainstem cysts (9 symptomatic cysts, 22 asymptomatic cysts), there was a 1.3 -fold difference $(p=0.5)$.

\section{Factors Associated With Cyst Burden, Development, and Size \\ Cyst Burden}

Increased cyst burden (total number of peritumoral cysts per patient) was associated with germline VHL mutation. Specifically, patients with a germline missense ( 0.7 cysts/patient) mutation had significantly fewer cysts than patients with partial deletions (1.1 cysts/patient; $\mathrm{p}=0.02)$ (Table 1).

\section{New Cyst Development}

Greater cyst burden at study entrance $(p=0.002)$ and younger age $(\mathrm{p}<0.0001,0.1$ cysts/year in patients younger than 35 years of age, or 1.1-fold compared with 0.06 cysts/ year if 35 years of age or older) were associated with an increased risk of new cyst development (Table 1).

\section{Cyst Size}

Cyst size at symptom development was dependent on location ( $\mathrm{p}<0.0001)$. Symptomatic cerebellum cysts were significantly $(\mathrm{p}<0.001)$ larger than symptom-causing spinal cysts (syringomyelia; $p=0.001,5.1$-fold), and/or brainstem cysts (syringobulbia; $\mathrm{p}=0.003,8.2$-fold) (mean cerebellum $3756 \mathrm{~mm}^{3}$ vs brainstem $456 \mathrm{~mm}^{3}$ and spine 730 $\mathrm{mm}^{3}$ ). There was no difference in size between symptomproducing spinal cysts and brainstem cysts $(\mathrm{p}=1.0)$. For asymptomatic cysts, there was no significant difference in size among the 3 locations (brainstem vs cerebellum, $\mathrm{p}=$ 0.8 ; brainstem vs spine, $\mathrm{p}=1.0$; cerebellum vs spine, $\mathrm{p}=$ 0.1) (Table 1).

\section{Factors That Predict Cyst-Associated Symptom Formation}

The ROC analysis demonstrated that cyst size thresholds in various anatomical regions can predict symptom formation (with area under ROC curve greater than 0.80). With a specificity threshold of $90 \%$ or more, cerebellar cysts attaining a diameter of $20.3 \mathrm{~mm}$ (an approximately $522.8 \mathrm{~mm}^{3}$ cyst volume), brainstem cysts attaining a diameter of $14.1 \mathrm{~mm}\left(175.2 \mathrm{~mm}^{3}\right.$ cyst volume), and spinal cord cysts attaining a diameter of $11.6 \mathrm{~mm}\left(97.6 \mathrm{~mm}^{3}\right.$ cyst volume) were predictably symptomatic.

\section{Surgical Management of Hemangioblastomas Associated With Cysts}

As detailed in our previous paper, ${ }^{11}$ there were 121 hemangioblastomas with associated peritumoral cysts (76.1\% of 159 tumors with associated peritumoral cysts) that were symptomatic and that required resection. Symptomatic tumors were located in the cerebellum (86 tumors; $54.1 \%$ of surgically treated tumors), brainstem $(9 ; 5.7 \%)$, spinal cord $(23 ; 14.4 \%)$, or supratentorial compartment $(1$; $0.6 \%$ ). All tumors were resected completely, and this universally resulted in collapse or resolution of the associated peritumoral cyst. The cyst wall was not removed in any case.
TABLE 1. Factors associated with cyst burden, development, growth, and size in patients with VHL disease

\begin{tabular}{lc}
\hline \multicolumn{1}{c}{ Factor } & p Value \\
\hline Cyst burden & \\
\hline Age in yrs $(\geq 35,<35)$ & 0.43 \\
\hline Yrs of follow-up & 0.04 \\
\hline Sex & 0.11 \\
\hline Mutation (partial deletion vs missense) & 0.02 \\
\hline Cyst development & \\
\hline Age in yrs $(\geq 35,<35)$ & $<0.0001$ \\
\hline No. of cysts at study entrance & 0.002 \\
\hline Sex & 0.05 \\
\hline Mutation (partial deletion vs missense) & 0.16 \\
\hline Cyst size & \\
\hline Location & $<0.0001$ \\
\hline Sex & 0.53 \\
\hline Mutation (partial deletion vs missense) & 0.64 \\
\hline Age in yrs $(\geq 35,<35)$ & 0.48 \\
\hline Years of follow-up (cyst) & 0.11 \\
\hline Tumor symptomatic type & $<0.0001$ \\
\hline Cyst growth rate & $<0.0001$ \\
\hline Location & 0.89 \\
\hline Sex & 0.94 \\
\hline Mutation (partial deletion vs missense) & 0.0006 \\
\hline Age in yrs $(\geq 35,<35)$ & $<0.0001$ \\
\hline Tumor symptomatic type & \\
\hline
\end{tabular}

\section{Discussion}

\section{Mechanisms of Peritumoral Cyst Formation}

Previous studies demonstrate that tumor-associated peritumoral cysts develop through a predictable series of steps..$^{10,14}$ Specifically, high intratumoral pressure and increased vascular permeability associated with hemangioblastomas, and the extremely high levels of vascular endothelial growth factor (originally known as vascular permeability factor $)^{3}$ that they produce, underlie plasma ultrafiltrate entering the surrounding interstitial spaces. Ultrafiltrate from circulating plasma flows from the increased interstitial pressure of tumor parenchyma into the surrounding parenchyma interstitium, producing local edema. Initially the tissue has the capacity to resorb the excess fluid. However, as time progresses (i.e., with increasing tumor size, increasing pressure differential, increasing permeability of the tumor vasculature, and/or chronic changes in the edematous tissue altering tissue capacity for resorption) and the ability of the tissue around the tumor to absorb the excess interstitial fluid is overcome, a cyst begins to form. The cyst will continue to grow until homeostasis between the rate of tumor plasma ultrafiltrate leakage and the rate of tissue resorption occurs.

\section{Previous Studies}

Previous studies have examined the presence of peritumoral cysts associated with hemangioblastomas in the 
CNS. ${ }^{1,14,26}$ These studies demonstrated that hemangioblastoma-associated peritumoral cysts are a frequent feature of hemangioblastomas when they occur sporadically or in patients with VHL disease. Moreover, these studies demonstrate that the presence of the peritumoral cyst and its associated mass effect can underlie the symptoms associated with CNS hemangioblastomas. Nevertheless, the previous studies were derived from retrospective analyses, and they did not examine the biological basis of peritumoral cysts.

To better understand the clinical impact and biology of hemangioblastoma-associated peritumoral cysts in an effort to improve management, we prospectively studied a large cohort of patients with VHL disease over long-term follow-up.

\section{Current Report}

\section{General Features}

Twelve percent of all VHL disease-associated CNS hemangioblastomas develop an associated peritumoral cyst. Nearly $60 \%$ of patients with VHL disease in the current study developed a hemangioblastoma-associated peritumoral cyst. Peritumoral-associated cysts were found in the cerebellum (63\% of total cyst burden of the CNS in patients with VHL disease), spinal cord (24\%), brainstem (11\%), and supratentorial compartment (1.4\%). Clinically, approximately $60 \%$ of cysts that were followed for more than 2 years progressed and caused symptoms requiring resection of the causative tumor. These findings underscore the critical impact that peritumoral cysts have on symptom formation and the need for surgical treatment.

\section{Imaging Features}

Consistent with previous data, ${ }^{10,14}$ peritumoral cysts form due to leakage of plasma ultrafiltrate through permeable tumor vessels that starts as edema. This biological feature is underscored by extravasation of gadolinium from the hemangioblastoma into the peritumoral cyst fluid on postcontrast FLAIR MRI. ${ }^{12}$ Because postcontrast FLAIR MRI is more sensitive to gadolinium in CSF and cyst fluid than in T1-weighted MRI, ${ }^{12}$ it is best for detecting this phenomenon. Further study may demonstrate that FLAIR MRI could permit qualitative (or quantitative) assessment of peritumoral fluid extravasation after pharmacological (e.g., steroid, vascular endothelial growth factor inhibitors, bevacizumab, or other similar agents) or other treatments (e.g., radiation).

T2-weighted and FLAIR MRI also can be used to track interstitial edema and cyst development and progression (Fig. 1). ${ }^{14}$ Although we did not quantitatively analyze T2weighted or FLAIR signal changes around cysts in the various regions of the nervous system, our experience and prior reports indicate that interstitial T2-weighted and FLAIR signal changes can decrease (or resolve) with cyst formation, progression, and/or stability. ${ }^{10,14}$ These imaging findings (i.e., frequent reduction/elimination of interstitial T2-weighted and/or FLAIR signal surrounding a peritumoral cyst) may be a result of the increased volumetric resorptive capacity afforded by a cyst (development or progression), and subsequent establishment of homeosta- sis between plasma ultrafiltrate production and/or leakage and cyst resorption.

\section{Cyst Distribution}

The majority of peritumoral cysts occur in the cerebellum (63\% of all cysts, and $14 \%$ of all cerebellar hemangioblastomas). This is substantially greater than the spinal cord $(24.3 \%$ of all cysts; $26 \%$ of spinal cord hemangioblastomas had an associated peritumoral cyst) and brainstem (11.3\% of cysts; $20 \%$ of brainstem hemangioblastoma had an associated peritumoral cyst), despite the fact that the overall distribution of CNS hemangioblastomas was equal between the cerebellum and spinal cord. The highest incidence of the occurrence of a cyst with a hemangioblastoma was in the brainstem and spinal cord. This may be due to differences in hydraulic conductivity of the spinal cord and brainstem compared with the cerebellum. These intrinsic anatomical differences in fluid transport may make the brainstem and spinal cord more prone to the generation of peritumoral cysts.

\section{Cyst Progression}

After peritumoral cyst formation was identified on MRI, cyst progression was a frequent finding. Half of all peritumoral cysts progressed within 1.5 years of observation, and nearly three-quarters $(72.3 \%)$ progressed within 3 years. Furthermore, $59 \%$ of cysts observed for more than 2 years had progressed enough to cause symptoms that required resection of the associated hemangioblastoma. Cyst progression was rapid when compared with tumor progression (50\% of tumors progressed in 5.6-8.9 years depending on tumor location, whereas $50 \%$ of cysts progressed in 1.5 years). Consistent with mass effect underlying symptomatology, the mean cerebellum cyst growth rate was 6 times faster than the mean associated tumor growth rate. Similarly, the ratio of cyst growth rate to tumor growth rate in symptomatic cysts in the cerebellum was more than 10 times faster than with asymptomatic cysts.

\section{Factors Affecting Burden, Development, and Size}

A greater number of peritumoral cysts at study entrance, younger age, and germline mutation were associated with increased risk of cyst development. Previous data demonstrate that VHL disease in those who harbor more hemangioblastomas at the study entrance, younger age, and partial deletion mutations have been shown to be associated with a greater number of hemangioblastomas, which could translate to more peritumoral cysts. ${ }^{11}$ Additionally, cysts causing symptoms in highly eloquent areas may occur at an earlier stage of progression. For example, cysts in the brainstem and spinal cord had symptoms, even though these were significantly smaller cysts than those in the cerebellum.

\section{Features Predictive of Symptom Formation}

Predictive analysis indicates that the size of the cyst based on anatomical location is an indicator of future symptom formation. Specifically, cerebellar cysts 20.3 $\mathrm{mm}$ or more in diameter (an approximately $522.8 \mathrm{~mm}^{3}$ cyst volume), brainstem cysts $14.1 \mathrm{~mm}$ or more in diameter (175.2 $\mathrm{mm}^{3}$ cyst volume), and spinal cord cysts 11.6 
$\mathrm{mm}$ or more in diameter $\left(97.6 \mathrm{~mm}^{3}\right.$ cyst volume) were associated with symptoms (specificity threshold of $90 \%$ or greater).

\section{Acknowledgment}

This research was supported by the Intramural Research Program of the National Institute of Neurological Disorders and Stroke at the National Institutes of Health.

\section{References}

1. Ammerman JM, Lonser RR, Dambrosia J, Butman JA, Oldfield EH: Long-term natural history of hemangioblastomas in patients with von Hippel-Lindau disease: implications for treatment. J Neurosurg 105:248-255, 2006

2. Asthagiri AR, Mehta GU, Zach L, Li X, Butman JA, Camphausen KA, et al: Prospective evaluation of radiosurgery for hemangioblastomas in von Hippel-Lindau disease. Neuro Oncol 12:80-86, 2010

3. Berkman RA, Merrill MJ, Reinhold WC, Monacci WT, Saxena A, Clark WC, et al: Expression of the vascular permeability factor/vascular endothelial growth factor gene in central nervous system neoplasms. J Clin Invest 91:153-159, 1993

4. Butman JA, Linehan WM, Lonser RR: Neurologic manifestations of von Hippel-Lindau disease. JAMA 300:1334-1342, 2008

5. Cockman ME, Masson N, Mole DR, Jaakkola P, Chang GW, Clifford SC, et al: Hypoxia inducible factor-alpha binding and ubiquitylation by the von Hippel-Lindau tumor suppressor protein. J Biol Chem 275:25733-25741, 2000

6. Filling-Katz MR, Choyke PL, Oldfield E, Charnas L, Patronas NJ, Glenn GM, et al: Central nervous system involvement in Von Hippel-Lindau disease. Neurology 41:41-46, 1991

7. Gläsker S: Central nervous system manifestations in VHL: genetics, pathology and clinical phenotypic features. Fam Cancer 4:37-42, 2005

8. Jagannathan J, Lonser RR, Smith R, DeVroom HL, Oldfield EH: Surgical management of cerebellar hemangioblastomas in patients with von Hippel-Lindau disease. J Neurosurg 108:210-222, 2008

9. Lamiell JM, Salazar FG, Hsia YE: von Hippel-Lindau disease affecting 43 members of a single kindred. Medicine (Baltimore) 68:1-29, 1989

10. Lohle PN, van Mameren H, Zwinderman KH, Teepen HL, Go KG, Wilmink JT: On the pathogenesis of brain tumour cysts: a volumetric study of tumour, oedema and cyst. Neuroradiology 42:639-642, 2000

11. Lonser RR, Butman JA, Huntoon K, Asthagiri AR, Wu T, Bakhtian KD, et al: Prospective natural history study of central nervous system hemangioblastomas in von HippelLindau disease. J Neurosurg 120:1055-1062, 2014

12. Lonser RR, Butman JA, Oldfield EH: Pathogenesis of tumorassociated syringomyelia demonstrated by peritumoral contrast material leakage. Case illustration. J Neurosurg Spine 4:426, 2006

13. Lonser RR, Glenn GM, Walther M, Chew EY, Libutti SK, Linehan WM, et al: von Hippel-Lindau disease. Lancet 361:2059-2067, 2003

14. Lonser RR, Vortmeyer AO, Butman JA, Glasker S, Finn MA, Ammerman JM, et al: Edema is a precursor to central nervous system peritumoral cyst formation. Ann Neurol 58:392-399, 2005

15. Lundin P, Pedersen F: Volume of pituitary macroadenomas: assessment by MRI. J Comput Assist Tomogr 16:519-528, 1992

16. Maddock IR, Moran A, Maher ER, Teare MD, Norman A,
Payne SJ, et al: A genetic register for von Hippel-Lindau disease. J Med Genet 33:120-127, 1996

17. Maher ER, Yates JR, Harries R, Benjamin C, Harris R, Moore AT, et al: Clinical features and natural history of von Hippel-Lindau disease. Q J Med 77:1151-1163, 1990

18. Mehta GU, Asthagiri AR, Bakhtian KD, Auh S, Oldfield EH, Lonser RR: Functional outcome after resection of spinal cord hemangioblastomas associated with von Hippel-Lindau disease. J Neurosurg Spine 12:233-242, 2010

19. Melmon KL, Rosen SW: Lindau's disease. Review of the literature and study of a large kindred. Am J Med 36:595-617, 1964

20. Neumann HP, Eggert HR, Scheremet R, Schumacher M, Mohadjer M, Wakhloo AK, et al: Central nervous system lesions in von Hippel-Lindau syndrome. J Neurol Neurosurg Psychiatry 55:898-901, 1992

21. Neumann HP, Lips CJ, Hsia YE, Zbar B: Von Hippel-Lindau syndrome. Brain Pathol 5:181-193, 1995

22. Richard S, Campello C, Taillandier L, Parker F, Resche F: Haemangioblastoma of the central nervous system in von Hippel-Lindau disease. J Intern Med 243:547-553, 1998

23. Richard S, Chauveau D, Chrétien Y, Beigelman C, Denys A, Fendler JP, et al: Renal lesions and pheochromocytoma in von Hippel-Lindau disease. Adv Nephrol Necker Hosp 23:1-27, 1994

24. Simone CB II, Lonser RR, Ondos J, Oldfield EH, Camphausen K, Simone NL: Infratentorial craniospinal irradiation for von Hippel-Lindau: a retrospective study supporting a new treatment for patients with CNS hemangioblastomas. Neuro Oncol 13:1030-1036, 2011

25. Stolle C, Glenn G, Zbar B, Humphrey JS, Choyke P, Walther $\mathrm{M}$, et al: Improved detection of germline mutations in the von Hippel-Lindau disease tumor suppressor gene. Hum Mutat 12:417-423, 1998

26. Wanebo JE, Lonser RR, Glenn GM, Oldfield EH: The natural history of hemangioblastomas of the central nervous system in patients with von Hippel-Lindau disease. J Neurosurg 98:82-94, 2003

27. Wind JJ, Bakhtian KD, Sweet JA, Mehta GU, Thawani JP, Asthagiri AR, et al: Long-term outcome after resection of brainstem hemangioblastomas in von Hippel-Lindau disease. J Neurosurg 114:1312-1318, 2011

28. Wind JJ, Lonser RR: Management of von Hippel-Lindau disease-associated CNS lesions. Expert Rev Neurother 11:1433-1441, 2011

\section{Disclosure}

The authors report no conflict of interest concerning the materials or methods used in this study or the findings specified in this paper.

\section{Author Contributions}

Conception and design: Lonser, Huntoon. Acquisition of data: Lonser, Huntoon, Oldfield. Analysis and interpretation of data: Lonser, Huntoon, Oldfield. Drafting the article: Lonser, Huntoon. Critically revising the article: Lonser, Huntoon, Oldfield. Reviewed submitted version of manuscript: Lonser, Huntoon, Elder, Butman, Chew, Linehan, Oldfield. Approved the final version of the manuscript on behalf of all authors: Lonser. Statistical analysis: $\mathrm{Wu}$.

\section{Correspondence}

Russell R. Lonser, Department of Neurological Surgery, The Ohio State University Wexner Medical Center, 410 W. 10th Ave., Doan Hall N1047, Columbus, OH 43210. email: russell. lonser@osumc.edu. 